\title{
Visual vigilance and brain damage: an empirical study
}

\author{
R. D. MCDONALD AND S. B. BURNS ${ }^{1}$ \\ From the Psychology Service of the Veterans Administration Hospital and the \\ Psychiatry Department of the Upstate Medical Center, Syracuse, New York, U.S.A.
}

Over the past 20 years a considerable amount of research has been expended on the use of human observers as detectors or monitors under conditions of monotony (Buckner and McGrath, 1963). In general these investigations have examined the capability of human senses for the detection of military targets as well as for quality control operations and automation in manufacturing. Such investigations are usually termed watch-keeping (Broadbent, 1953) or vigilance (Mackworth, 1950) experiments. The latter term is based upon the concept of the physiological adaptive efficiency of the central nervous system (Head, 1926). Specifically, vigilance research deals with the attentiveness of the subject and his capacity for selectively detecting and appropriately responding to changes in stimulus events over relatively long periods of time. A detection task is involved, i.e., a task in which the subject is required to indicate the presence or absence of some specified change (critical signal) in his operating environment. Performance (signal detection) deteriorates progressively as a function of time-onwatch with either visual or auditory modalities (Adams, 1956; Mackworth, 1950; Monty, Myers, and Murphy, 1958). Variables effective in alleviating this performance decrement are summarized in great detail elsewhere (McGrath, Harabedian, and Buckner, 1959), and a comprehensive review of the major theoretical ad hoc positions in the area is offered by Frankmann and Adams (1962). It is to be noted that none of these positions account for any substantial part of the empirical data.

Within the watch-keeping framework, the term 'vigilance' has been used to refer to a central process or state of the organism (Bakan, 1952; Mackworth, 1957). There are several reasons for assuming that the phenomena obtained in vigilance studies are central in origin (McGrath, Harabedian, and Buckner, 1959; McGrath, 1963). 1 Performance changes are not restricted to a single criterion measure but are somewhat general; 2 a number of

IU.S. National Institute of Mental Health Fellow. different purely psychological variables affect performance levels; 3 time per se is of course not causal.

If the progressive deterioration in detecting signalse commonly reported is indeed central in origin, it $\overrightarrow{0}$ would seem a reasonable $a$ priori assumption that the monitoring performance of brain-damaged $\vec{\omega}$ individuals would differ from that of individuals: not so affected. It is somewhat surprising that no systematic studies of individuals with insults to thei central nervous system have been carried out $t_{0}^{\mathrm{w}}$ evaluate this assumption (Mackworth, 1959; Mondiv 1963).

The object of this paper is to report an empirieate investigation of the effects of intracranial orgapic pathology on monitoring performance.

\section{METHOD}

SUBJECTS Six independent groups of 10 male hunfing subjects were used: two of brain-damaged subjects, twa $a^{+}$ of hospital controls, and two of normal controls. Patients from the Syracuse Veterans Hospital and the Onondagas County Hospital made up the first four groups. For these groups independent neurological or psychiatric opinion as to the presence or absence of supratentorial insult, classi-용 fication as non-psychotic, and the ability to perform the task was obtained. Particular attention was given to verify $\vec{\sigma}$ the absence of defects of visual field and eye movement. 3 A variety of anatomical and aetiological diagnoses was represented in the brain-damaged groups. The hospitat control groups were composed primarily of patients: suffering from fractures of the lower limbs. The norma $\overline{5}$ controls were drawn from a heterogeneous population of older college students and white and blue collar workers All subjects were volunteers.

No attempts were made to equate the groups for chronological age, race, or education. Mean ages for the brain-damaged groups were 52.0 years (S.D. $=19.7$ ) andb 53.7 (S.D. $=14.8)$, for hospital controls $52 \cdot 1$ (S.D. $=15 \cdot 8)$ and $52 \cdot 1$ (S.D. $=17 \cdot 9)$, and for the normal controls $27 \cdot 7$ $($ S.D. $=12 \cdot 4)$ and 26.0 (S.D. $=12 \cdot 2)$. Nineteen additionaE. younger hospital controls were tested and their perform-N ance, together with that of the other hospital controls; plotted as a function of age. No relationship between performance and age was seen. 
APPARATUS The visual display was a Marion decibel meter (6 MW 0 level 500 ohms) set in a flat black wooden panel at eye level and about 3 feet from the subject. The meter has a circular face about $2 \frac{1}{4}$ in. in diameter and a pointer about $1 \frac{3}{4}$ in. long. This display closely resembled that used by Monty (1962). Regular deflections of the meter from the null or far-left position to a mid-dial position were introduced automatically about once a second. The signal to be detected by the subject was a deflection that exceeded the mid-dial position by about $\frac{5}{16}$ in. A telegraph key mounted on a movable lapboard served as the response key. All subjects performed the task seated in a wheelchair, as many were not ambulatory. Depressions of the telegraph key were displayed linearly on a paper puller (event recorder). Test conditions of relatively constant illumination were obtained by using artificial background illumination. To reduce auditory distraction continuous white noise (16 VU) was present throughout the experiment.

PROCEDURE Subjects were brought to the experimental room, seated in the wheelchair, the lapboard with the telegraph key placed over the wheelchair arms and the instructions given. The subject was instructed to press the telegraph key with his preferred hand whenever the pointer deflected beyond the mid-dial position, i.e., upon detection of a critical signal. Before the experimenter left the room the subject was required to detect three practice critical signals. Each subject attended only one experimental session of 50 to $60 \mathrm{~min}$. and performed under one of two rates of critical signal presentation, high or low. In the high rate 60 critical signals per hour were presented, in the low 12. These presentations were programmed so that for both high and low rates an equal percentage of critical signals occurred within successive 15 -min. time blocks. The design of this experiment then was a groups by critical signal rate by time periods, i.e., a $3 \times 2 \times 3$ factorial with the third factor (time) dependent.

A preliminary study with 20 male undergraduate students from Syracuse University (10 students per critical signal rate) indicated a progressive decrement in critical signal detection over time and a larger overall percentage missed under the low rate condition than under the high. Therefore, it was concluded that the conditions of this experiment were comparable to those reported by Monty (1962).

\section{RESULTS}

The percentage of critical signals missed (percentage error) for each of the first three 15-min. intervals was obtained for each subject by dividing the number of times the subject failed to press the telegraph key during any 15 -min. interval by the number of critical signals presented during that interval. This percentage conversion allowed meaningful comparisons between groups performing under the two different critical signal rate conditions.

Mean percentage error is shown in Table I. Inspection of Table I shows for all groups (1) a progressive increment in percentage of critical signals missed over time, (2) a greater percentage error for the low signal rate condition than for the high, and (3) an ordering effect, the brain-damaged subjects missing more critical signals than the hospital controls who in turn missed more than the normal controls. A $2 \times 3 \times 3$ variance analysis statistically supported the preceding statements made from an examination of Table I. Significant differences were found between (1) time periods ( $F$ with 2, 108 d.f. $=18.24, p<0.001)$, (2) critical signal rates $(F$ with 1,54 d.f. $=4.80, \mathrm{p}<0.05)$, and (3) groups (F with 2,54 d.f. $=7.90, \mathrm{p}<0.01)$. None of the interaction terms were statistically significant.

\section{TABLE I}

MEAN PERCENTAGE CRITICAL SIGNALS MISSED AS A FUNCTION OF 15-MIN. PERIODS AND SIGNAL RATE

Successive 15-Min. Periods

\begin{tabular}{llll}
\cline { 3 - 4 } Group (10 Subjects per Group) & $I$ & $I I$ & $I I I$ \\
\hline Low Critical Signal Rate & & & \\
Brain-damaged & 43.0 & $73 \cdot 0$ & $73 \cdot 2$ \\
Hospital control & 26.5 & $46 \cdot 2$ & $56 \cdot 3$ \\
Normal control & $19 \cdot 8$ & $23 \cdot 2$ & $39 \cdot 9$ \\
High Critical Signal Rate & & & \\
Brain-damaged & 39.9 & $45 \cdot 2$ & $54 \cdot 0$ \\
Hospital control & $15 \cdot 1$ & $23 \cdot 2$ & 31.9 \\
Normal control & 6.0 & $22 \cdot 7$ & 29.9
\end{tabular}

To determine overall significance of performance differences over time periods within a group for a particular critical signal condition, simple trend analyses of variance were performed. For the low critical signal programme statistically significant increments in percentage of critical signals missed were found for the brain-damaged subjects ( $F$ with 2 , 18 d.f. $=4.36, \mathrm{p}<0.05)$ and hospital controls $(\mathrm{F}$ with 2,18 d.f. $=4 \cdot 31, p<0.05$ ). However, for the normal controls performance was comparable across time periods in the low programme condition $(F$ with 2,18 d.f. $=1 \cdot 57, \mathrm{p}<0 \cdot 10$ ). For the high critical signal programme statistically significant increments in percentage of critical signals missed were found for the brain-damaged subjects ( $F$ with 2,18 d.f. $=3 \cdot 84$, $\mathrm{p}<0.05$ ) and for the normal controls (F with 2,18 d.f. $=7 \cdot 13, p<0.01)$. However, for the hospital controls performance was comparable across time periods in the high programme condition ( $\mathrm{F}$ with 2,18 d.f. $=3.01, \mathrm{p}<0.05$ ).

Specific comparisons of performance differences between the two critical signal rate conditions (low versus high) within groups were made by $t$ tests. The brain-damaged subjects missed a significantly greater percentage of critical signals in the low rate condition than in the high ( $\mathrm{t}$ with 58 d.f. $=2.66$, $p<0.05)$ as did the hospital controls ( $t$ with 58 d.f. $=3.75, p<0.01$ ). However, performance between the two rate conditions for the normal controls 
was comparable ( $\mathrm{t}$ with 58 d.f. $=1 \cdot 46, \mathrm{p}>0 \cdot 10$ ).

Within a particular critical signal rate condition (low or high) performance comparisons between groups were made by $t$ tests. In the low rate condition the brain-damaged subjects missed a significantly greater percentage of critical signals than either the hospital controls ( $t$ with 58 d.f. $=3.18, p<0.01$ ) or the normal controls ( $\mathrm{t}$ with 58 d.f. $=6.53, \mathrm{p}<0.01$ ). Likewise, in the low rate condition the hospital controls missed a significantly greater percentage of critical signals than the normal controls ( $t$ with $\mathbf{5 8}$ d.f. $=2.43, \mathrm{p}<0.05)$. In the high rate condition a similar result was obtained, i.e., the brain-damaged subjects missed significantly more critical signals than the hospital controls ( $\mathrm{t}$ with 58 d.f. $=4.43$, $\mathrm{p}<0.01$ ) or the normal controls ( $\mathrm{t}$ with $58 \mathrm{~d} . \mathrm{f}$. $=$ $5 \cdot 18, p<0.01)$. However, in the high rate condition the performance of the hospital controls was comparable to that of the normal controls ( $t$ with 58 d.f. $=0.93$ ).

Errors of commission (pressing the telegraph key in the absence of a critical signal) were so infrequent as to make statistical analysis of little value. Numbers of such errors were comparable between groups in a particular rate programme. In general these errors of commission were slightly more frequent in the higher critical signal rate condition.

\section{DISCUSSION}

TASK ADEQUACY The results of the statistical analyses, together with the subjective reports of the subjects, seem to indicate that the desired effects were obtained, that is, the subjects were placed in a monotonous monitoring situation which led to a decrement in performance in the number of critical signals detected as a function of time. In addition, degree of deterioration in performance was found to be a function of signal rate. The more frequent the critical signals, the less the deterioration in performance. These observations are in keeping with the general literature (Buckner and McGrath, 1963). While there was no known instance of a subject falling asleep, many reported that they never thought 'they'd make it' to the end of the session. Indeed, a most common occurrence was the overt verbalization (singing, chanting) of the subject during the session.

SUSTAINED PERFORMANCE The lack of statistical significance of any of the interaction terms in the analysis indicates within the time period studied that the overall performance curves were of comparable shape. That is, in this monitoring situation the braindamaged subjects showed the same rate of performance decrement as did those not so injured. Differences between these classifications were one of degree, not of kind. These data would seem to contradict the hypothesis of diminished capacity for work proposed by Goldstein (1942) that braing damaged individuals differ from those not so in jured in sustained levels of performance.

CONTROL SUBJECTS The reliable performance differ ence found between the hospital and normal controls in the low rate condition and the lack of such $\frac{5}{8}$. difference in the high rate condition point ou methodological cautions in terms of applying generalized conclusions to a non-hospitalized populas? tion from studies which use only hospitalized contred subjects. The question of appropriate control groups is raised, or, more specifically, the question of the effects of hospitalization per se. Assuming the ag factor to be unimportant, if the high rate programme is considered a relatively easy task with respect to the low, then a reasonable explanation would be tha hospitalization factors manifest themselves only on more difficult tasks. Such an explanation is consistenf with recent data in the area of simple reaction times with age controlled. Kushner (1963) reported n₹ difference between hospitalized and non-hospitalize subjects in what would be construed an easy taखis while McDonald (1963) noted the significantif inferior performance of hospitalized individualspas

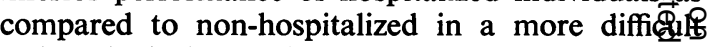
task. A logical extension of this effect of hospitalization with respect to this easy/difficult dichotomb is in terms of a lower/higher order construct-dictọ tomy.

BRAIN-DAMAGED SUBJECTS Based upon neurologicait opinion, the brain-damaged subjects were sorted into three general anatomical categories: 1 brain-sten vascular accidents (excluding the cerebellum), ( $2 \Phi$ insult to the basal ganglia, and (3) diffuse cerebra disease. In the low critical signal rate condition n\&्Q performance trend with respect to anatomical cate $\vec{\sigma}$ gory was seen. The mean percentage error of the subjects in each category, 1 , three subjects, 2, three? and, 3, four, respectively clustered rather tighti. around the overall mean. However, in the high critical signal rate an interesting trend was obtained the four subjects with insult to the basal ganglie made three times as many errors as did the others. (1, two subjects and, 3 , four). It is, of course, dangero ous to generalize from the performance of so fev subjects. However, the intriguing speculation ariseg of a very simple performance task which differen tiates individuals with involvement of the basa. ganglia. It is to be noted that such obtained per formance decrement is not due to a motor incapacity, that is, it can be due neither to a delay in key pressind nor to too many key presses. In this task speed per sॄ్ట 
is irrelevant. Secondly, the number of covert responses (pressing the key when no critical signal appeared) was no different for these basal ganglia subjects than for the other brain-damaged subjects or the various controls. The differentiation in performance of the subjects with injuries to the basal ganglia in the high rate condition as opposed to the lack of such a differentiation in the low may reflect an arithmetical artifact. That is, in the low condition relatively few correct detection responses were possible while in the high a greater number are available. This relative ratio of possible errors would make data in the high rate condition more reliable.

\section{SUMMARY}

The effect of brain damage on visual monitoring performance at two rates of critical signal presentation (low and high) was empirically investigated. Six independent groups of 10 male subjects were used: two of hospitalized brain-damaged patients (one at each rate of signal presentation), two hospitalized subjects without brain damage, and two of normal controls. In general, the data support the following statements: 1 Detection performance of the brain-damaged subjects was inferior to that of the controls; 2 deterioration in performance was greater in the low rate condition than in the high, and, 3, detection of critical signals decreased as a function of time.

The question of appropriate control groups was raised as well as the notion that this monitoring task might serve as a possible indicator of insult to the basal ganglia.
It was concluded that these data are consistent with the use of the term 'vigilance' as referring to a central process or state of the organism.

We should like to thank Drs. Anthony T. Ladd and James MacD. Watson (Syracuse Veterans Administration Hospital) and Drs. Herbert Notkin and Kenneth Wright (Onondaga County Hospital) who, together with their staffs, provided the hospitalized patients. Our thanks are also due to Dr. R. A. Monty (Cornell Aeronautical Laboratory, Inc.) who critically read this manuscript.

\section{REFERENCES}

Adams, J. A. (1956). J. exp. Psychol., 52, 204.

Bakan, P. (1952). Univ. Ill. Training Res. Lab. Memo. Rep., no. 8-1.) Broadbent, D. E. (1953). Psychol. Rev., 60, 331.

Buckner, D. N., and McGrath, J. J. (1963). Vigilance: A symposium. McGraw-Hill, New York.

Frankmann, J. P., and Adams, J. A. (1962). Psychol. Bull., 59, 257.

Goldstein, K. (1942). After Effects of Brain Injuries in War. Grune and Stratton, New York.

Head, H. (1926). Aphasia and Kindred Disorders of Speech. University Press, Cambridge.

Kushner, E. N. (1963). Percept. Mot. Skills, 17, 321.

Mackworth, N. H. (1950). Spec. Rep. Ser. med. Res. Coun. (Lond.), No. 268.

- (1957). Advanc. Sci., 13, 389

(1959). Personal communication.

McDonald, R. D. (1964). J. nerv. ment. Dis., 138, 241-247.

McGrath, J. J., Harabedian, A., and Buckner, D. N. (1959). Hum. Factors Res. Inc. Tech. Report, no. 1 .

(1963). Some Problems of Definition and Criteria in the Study of Vigilance Performance. In Vigilance: $A$ Symposium. Edited by D. N. Buckner, and J. J. McGrath, pp. 227-246. McGraw-Hill, New York.

Monty, R. A. (1962). Hum. Factors, 4, 201

- (1963). Personal communication.

-_, Myers, T. I., and Murphy, D. B. (1958). U.S. Army Leadership Human Research Unit Res. Paper, no. 22. 